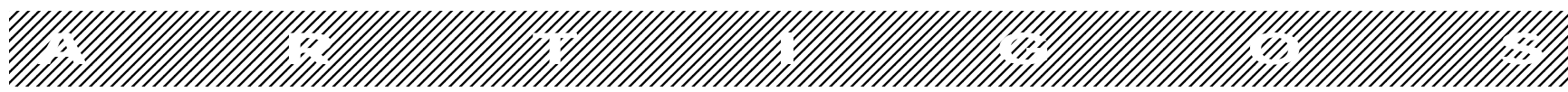

\title{
Otimismo comparativo e percepções de controle face à saúde na adolescência: existirão diferenças etárias?
}

\author{
Sónia F. Bernardes \\ Maria Luísa Lima \\ Instituto Superior das Ciências do Trabalho e da Empresa (Portugal)
}

\begin{abstract}
Resumo
O Otimismo Comparativo (OC), a tendência da maioria das pessoas para ver o seu futuro como mais positivo que o futuro dos outros, e a sua relação com a Percepção de Controle estão pouco estudados na adolescência. Os objetivos deste estudo prenderam-se com a análise das diferenças etárias na adolescência: (1) no OC e Percepções Comparativas de Controle (PCC) face a riscos para a saúde e (2) na forma como as Percepções Absolutas e Comparativas de Controle predizem o OC. Noventa e um pré-adolescentes ( $M=11,92$ anos) e 97 adolescentes ( $M=16,86$ anos) saudáveis efectuaram julgamentos de risco e controle (Próprio vs. Alvo) para 8 enfermidades. Os participantes mostraram-se comparativamente otimistas, julgando ter maior controle sobre algumas doenças que o alvo. Não existiram diferenças etárias no OC ou nas PCCs. Estas foram o principal preditor do OC, mediando a relação entre este e as Percepções Absolutas de Controle apenas na subamostra dos adolescentes.
\end{abstract}

Palavras-chave: otimismo comparativo; percepção de controle; adolescência; saúde

\begin{abstract}
Comparative optimism and perceived control over health in adolescence: are there any age differences? Comparative Optimism (CO) is the tendency for most people to perceive their future as rosier than the future of others. Such optimistic bias and its relationship with Perceived Control have seldom been studied among adolescents. Hence, this study aimed at exploring age differences throughout adolescence in: 1) CO and Comparative Perceived Control (CPC) over health hazards; 2) the way Absolute and Comparative Perceived Control account for CO. Ninety-one pre-adolescents $(M=11.92$ years) and 97 adolescents $(M=$ 16.86 years), all considered healthy, were asked for risk and control judgements (Self vs. Target) regarding 8 health hazards. Generally, participants were comparatively optimistic and saw themselves as having more personal control over some hazards than the target. No age differences were found in CO or CPC. The latter was the best predictor of $\mathrm{CO}$ for both cohorts. It mediated the relationship between Absolute Perceived Control and CO in the older participants' sample.
\end{abstract}

Key words: comparative optimism; perceived control; adolescence; health

$\mathrm{O}$ Otimismo Comparativo (OC) é a tendência da maioria das pessoas para julgar o seu futuro como mais positivo que o futuro de outros (Armor \& Taylor, 1998; Weinstein, 1980; Weinstein \& Klein, 1996). Nas últimas décadas, têm-se constatado a sua presença face a uma enorme variedade de riscos para a saúde. Hipertensão, úlceras, bronquites, cefaléias, obesidade, anorexia, sífilis, AIDS, gonorréia, ataque cardíaco, câncer, esquizofrenia, são apenas alguns exemplos de enfermidades que na perspectiva da maioria das pessoas assolarão com maior probabilidade o futu- ro de outros (Chapin, 2000; Clark, Williams \& Arthey, 1997; Fontaine \& Smith, 1995; Harris \& Middleton, 1994; Van der Velde, Hooykaas, \& Van der Pligt, 1991; Weinstein, 1980, 1987).

Muitos investigadores se têm debruçado sobre o papel aparentemente controverso que o OC parece desempenhar na saúde física e mental dos indivíduos. Por um lado, existe uma linha de investigação que aponta para o fato de o OC, ao minimizar a percepção de susceptibilidade face a riscos, poder afetar negativamente os esforços de prevenção. De fato, evidências mostram que as pessoas que se percebem como 
mais invulneráveis a riscos têm menor probabilidade de usar contraceptivos (Burger \& Burns, 1988), de se protegerem de relações sexuais de risco (Sheer \& Cline, 1994), de não usarem cintos de segurança ou de conduzirem em excesso de velocidade (McKenna, Stainer, \& Lewis, 1991). Por outro lado, muitos estudos têm enfatizado os benefícios de tal enviesamento, particularmente em nível dos processos de adaptação a situações de doença (Armor \& Taylor, 1998). O OC parece fomentar comportamentos de prevenção e estratégias ativas de coping entre soropositivos (Taylor et al., 1992), ou mesmo, ter impacto positivo no funcionamento do sistema imunológico dos mesmos (Segerstrom, Taylor, Kemeny, \& Fahey, 1998).

Apesar do grande volume de literatura que se debruça sobre esta temática, um leitor mais atento poderá facilmente constatar que tais evidências têm sido encontradas majoritariamente em amostras de adultos. Aparentemente, ainda pouco se sabe sobre a forma como crianças e adolescentes entendem os riscos que os rodeiam. A possibilidade da existência de diferenças na expressão do OC em indivíduos que se encontrem em diferentes momentos do seu desenvolvimento parece ser uma questão fundamental e pouco clarificada.

Atualmente, desde cedo se verifica um esforço por parte das instituições escolares para desenvolver programas de prevenção primária que visam alertar crianças e adolescentes para diversos riscos de saúde, com a intenção da implementação e generalização de hábitos comportamentais de prevenção. Estes programas parecem ser concebidos, todavia, sem existir uma noção apurada da forma como crianças e adolescentes de diferentes grupos etários entendem tais riscos, o que poderá colocar em cheque a eficácia de tais esforços educativos.

É apenas na última década que se encontram alguns estudos com faixas etárias mais jovens, explorando o OC no contexto da saúde. Tais estudos são efetuados majoritariamente com adolescentes, denotando uma grande preocupação pela compreensão de tal fenômeno nesta fase específica do desenvolvimento. A adolescência é vista como uma fase de experimentação que, associada a um aumento gradual de autonomia, parece colocar o adolescente mais susceptível a comportamentos de risco que poderão ter conseqüências graves para a sua saúde. Esta problemática tem vindo a ser considerada por muitos uma questão central de saúde pública e, como tal, a sua compreensão poderá acarretar uma série de implicações práticas a nível educacional, social e legal (Beyth-Marom, Austin, Fischhoff, Palmgreen, \& Quadrel, 1993; Millstein, 1993; Quadrel, Fischhoff, \& Davis, 1993).

A percepção de invulnerabilidade freqüentemente associada à adolescência tem sido uma das principais hipóteses levantadas para explicar a elevada freqüência de comportamentos de risco nesta etapa do desenvolvimento. Uma das teorizações centrais a este respeito é a de Elkind (1967), que aponta para o fato de tais percepções de invulnerabilidade serem subprodutos de um egocentrismo típico da adolescência, conseqüência inevitável do processo de desenvolvimento cognitivo. Segundo este autor, um pensamento egocêntrico iria ter como conseqüência, entre outras, o fenômeno da fábula pessoal. Este diz respeito à crença que os adolescentes possuem de que são únicos, de que mais ninguém no mundo poderá compreender o que pensam e o que sentem. Esta percepção de individualidade e unicidade poderá ser tão extrema que "se torne numa conviç̧ão de que não vai morrer, de que a morte acontecerá a outros, mas não a ele” (Elkind, 1967, p.1031). De fato, evidências empíricas recentes vêm suportar a relação positiva entre a presença de crenças de fábula pessoal e percepções de invulnerabilidade comparativa na adolescência, especificamente em relação a ser vítima de violência (Chapin, de las Alas, \& Coleman, 2005). Todavia, apesar desta teoria ser uma das mais citadas, apontando para um pico na percepção de invulnerabilidade ao risco na adolescência, é uma hipótese fundamentalmente assente em evidências clínicas e que, atualmente, apresenta escassas evidências empíricas que a suportem (Cohn, Macfarlane, Yanez, \& Imai, 1995; Millstein, 1993; Quadrel et al., 1993).

Analisando a pouca evidência disponível verifica-se sistematicamente que os adolescentes demonstram possuir, à semelhança dos adultos, enviesamento otimista. Estes resultados verificam-se para uma série de eventos tais como a toxicodependência, aids, tabagismo, acidente de aviação, câncer, ataque cardíaco, alcoolismo ou ser vítima de violência (Arnett, 2000; Chapin, 2001; Chapin et al., 2005; Chapin \& Gleason, 2004; Cohn et al., 1995; Gladis, Michela, Walter, \& Vaughan, 1992; Kershaw et al., 2003; Quadrel et al., 1993; Whalen et al., 1994). No entanto, os estudos que visam fazer a comparação da percepção de invulnerabilidade entre adolescentes e adultos parecem não apoiar de forma unânime a hipótese de Elkind. Apesar de algumas evidências apresentarem os adolescentes como mais otimistas que os adultos face à dependência do tabaco ou às suas conseqüências nefastas (Arnett, 2000), outros dados apontam, por sua vez, para a inexistência de diferenças no OC (Chapin, 2001), parecendo mesmo existir uma ligeira tendência para adolescentes se perceberem como mais vulneráveis que os próprios pais a acidentes de carro, gravidez não planejada (Quadrel et al., 1993) e diferentes tipos de ferimentos e doenças (Cohn et al., 1995).

A escassa literatura aqui mencionada referente ao contexto da saúde apresenta, do nosso ponto de vista, algumas limitações. Ao fazerem a comparação do OC entre adolescentes e adultos, os autores parecem definir os limites etários da adolescência de forma um pouco vaga, podendo estar incluídos no mesmo grupo participantes dos 11 aos 18 anos. Apenas Cohn e colaboradores (1995) fazem uma breve referência à inexistência de efeitos da idade, embora não esteja clara a operacionalização dos grupos etários. Já Whalen e colaboradores (1994), apesar de definirem de forma explícita e teoricamente fundamentada a escolha de um grupo etário específico (dos 10 aos 13 anos), não conferem um caráter comparativo ao seu estudo, sendo apenas possível analisar 
o fenômeno do OC para aquele grupo em particular. Finalmente é de salientar a escassez de estudos com pré-adolescentes.

$O$ estudo discriminado das diferentes fases da adolescência é tanto mais importante quando os estudos desenvolvimentistas sobre as conceptualizações de saúde e doença apontam para a possibilidade de tais significações sofrerem grandes alterações ao longo deste período de desenvolvimento (e.g., Barrio-Martinez, 1990; Perrin \& Gerrity, 1981). Na pré-adolescência (10/12 anos), a proximidade das operações concretas reflete-se com freqüência numa representação de doença assente nos seus aspectos perceptivos, que resulta da ação de agentes externos (e.g., microrganismos) que invadem o organismo através de processos de internalização que, a par dos mecanismos internos de produção da doença, ainda não são inteiramente compreendidos. Já para o final da adolescência, e com o advento das operações formais, a doença passa a ser vista como um processo psicofisiológico complexo, existindo um reconhecimento da causalidade múltipla bem como do papel ativo do próprio organismo na produção e combate da doença. A possibilidade de as diferentes conceptualizações de saúde e doença poderem trazer consigo diferenças em nível das percepções de risco face à saúde, leva-nos a enfatizar a importância do estudo do OC de forma diferenciada ao longo deste período de desenvolvimento. Assim, é com este intuito que o presente estudo pretende ter como primeiro objetivo geral o da análise das diferenças etárias ao longo da adolescência no enviesamento otimista face a riscos para a saúde.

Todavia, para além da análise da existência de OC ao longo da adolescência, linhas de investigação recentes têm enfatizado a importância do estudo dos seus fatores determinantes. Mais do que mostrar a existência de tal enviesamento é importante delimitar quais os fatores que formatam a sua expressão (Helweg-Larsen \& Shepperd, 2001).

Um dos fatores que mais tem sido estudado e cuja relação com tal fenômeno se tem mostrado um dado empírico robusto é a Percepção de Controle. Um dos primeiros autores a hipotetizar e comprovar empiricamente tal relação foi Weinstein (1980), mostrando estar a intensidade do OC fortemente relacionada com o grau em que os sujeitos percebem os riscos como controláveis. Nos últimos anos, um vasto corpo de literatura tem apoiado tal relação (Harris, 1996; Harris \& Middleton, 1994; Helweg-Larsen \& Shepperd, 2001; Kos \& Clarke, 2001; McKenna, 1993; Moen \& Rundmo, 2005; Taylor et al., 1992; Van der Velde et al., 1991). Uma recente meta-análise de 22 estudos mostra que o tamanho do efeito da percepção de controle sobre o OC é considerável ( $r=0,31$; Klein \& Helweg-Larsen, 2002). Aliás, pensa-se que o efeito sistemático da experiência pessoal com eventos negativos na redução da percepção de invulnerabilidade comparativa (e.g., McKenna \& Albery, 2001; Weinstein, 1980) se deve em parte a uma percepção de perda pessoal de controle sobre tais eventos (Helweg-Larsen \& Shepperd, 2001). Embora escassos, alguns estudos empíricos também têm mostrado esta relação para amostras de adolescentes e não apenas de adultos (Chapin et al., 2005; Quadrel et al., 1993; Whalen et al., 1994).

Apesar de todas as evidências, a relação entre a percepção de controle e o OC não é imediatamente evidente. É certo que quando um acontecimento é percebido como controlável, implica que o indivíduo consiga conceber a existência de uma seqüência de ações que, eventualmente, aumentará a probabilidade de um resultado desejado ou diminuirá a probabilidade de um resultado indesejado (Harris, 1996; Weinstein, 1980). No entanto, embora seja lógica a relação entre a percepção de controle e o julgamento absoluto sobre a probabilidade de ocorrência de um evento, não é imediatamente compreensível a razão pela qual a noção subjetiva de controle sobre um evento possa estar associada ao fato de o sujeito acreditar estar mais invulnerável a determinados riscos em comparação com outros. Teoricamente, esta relação só ganha sentido se se assumir que as próprias percepções de controle podem elas próprias ser enviesadas (Harris, 1996; Harris \& Middleton, 1994).

Recentemente, Thompson e colaboradores (1998) vêm defender a teoria de que os julgamentos de controle assentam na utilização da chamada Heurística do Controle. Segundo estes autores, para que um indivíduo tenha a percepção de controle sobre uma situação ou acontecimento, basta encontrar pistas que apontem, ora para a relação entre as suas próprias ações e um determinado resultado, ora para a intencionalidade na obtenção do mesmo. Dado que tanto os julgamentos de intencionalidade como os julgamentos de contingencialidade são eles próprios cheios de falhas e enviesamentos, é relativamente freqüente que os sujeitos julguem ter mais controle sobre determinadas situações ou acontecimentos que aquele que na realidade possuem. Se juntamente com esta hipótese, considerarmos a possibilidade de existir uma tendência egocêntrica por parte dos indivíduos para desconsiderar a possível relação entre as ações dos outros e determinados resultados, ou mesmo, para acreditar na maior eficácia das suas próprias ações (Weinstein, 1982), então não é senão natural considerarmos que os julgamentos de controle têm grande probabilidade de serem eles mesmos comparativamente enviesados.

Efetivamente, existem algumas evidências empíricas que apontam para o fato de os indivíduos se julgarem superiores a outros numa série de dimensões relacionadas com o controle, tais como, a sua posição face a determinados fatores de risco (e.g., Redmond \& Griffith, 2004) ou mesmo os comportamentos de prevenção que possuem no seu repertório (Harris, 1996). Mais ainda, alguns estudos têm mostrado que os indivíduos que efetuam julgamentos de risco comparativamente otimistas tendem a perceber-se como tendo mais controle, em diferentes dimensões, que o alvo em questão. Embora Harris e Middleton (1994) não tenham encontrado uma relação direta entre os julgamentos comparativos de controle e de risco face a uma série de eventos negativos para a saúde verificam que, de uma maneira geral, os indivíduos com maior OC se consideram simultaneamente, como tendo um menor número de 
comportamentos de risco e possuindo um maior número de atributos saudáveis que os alvos de comparação. Quadrel e colaboradores (1993) vêm mostrar existir, tanto para adultos como para adolescentes, uma correlação positiva bastante significativa entre o OC e as percepções comparativas de controle sobre uma série de eventos que afetam a saúde dos indivíduos.

Apesar de tais evidências, continuam a ser raros os estudos que procuram analisar as percepções de controle de forma comparativa; particularmente, durante a adolescência. Mais ainda, raramente se procuraram testar e diferenciar os efeitos preditivos dos julgamentos absolutos e comparativos de controle sobre o OC, dado que a maioria das investigações se centram apenas nas percepções absolutas de controle. Por este motivo, para além da análise das diferenças etárias no OC, procuramos ainda analisá-las (2) nas percepções comparativas de controle dos jovens adolescentes sobre diversos riscos face à saúde e (3) no valor preditivo das percepções absolutas e comparativas de controle sobre o OC face à saúde.

\section{Método}

\section{Participantes}

Participaram 188 indivíduos no presente estudo. Noventa e um eram pré-adolescentes a freqüentar o correspondente no Brasil à 6 ${ }^{\underline{a}}$ série da $2^{\text {a }}$ fase do ensino fundamental (idades compreendidas entre 11,33 e 13,33 anos; $M=11,92$; $D P=$ $0,43)$. Noventa e sete eram adolescentes a freqüentar o correspondente no Brasil ao 2oano do ensino médio (idades compreendidas entre 15,83 e 17,83 anos; $M=16,86$; $D P=$ 0,41). Todos os participantes freqüentavam escolas públicas da área metropolitana da Grande Lisboa. Verificou-se uma distribuição equilibrada dos indivíduos por sexo, tanto para os pré-adolescentes ( $n=41$ e 50 para meninos e meninas, respectivamente), como para os adolescentes ( $n=50$ e 47 para rapazes e moças, respectivamente). A análise do nível de escolaridade dos progenitores permitiu constatar que a grande maioria dos pais e das mães possuía um nível de escolaridade igual ou superior ao correspondente no Brasil ao 3o ano do ensino médio (76\% e 74,9\% respectivamente). Verificou-se, no entanto, que a amostra dos pré-adolescentes possuía uma maior percentagem de pais e mães com um nível de escolaridade igual ou inferior à 9a série da $2^{\underline{a}}$ fase sendo, simultaneamente, menor a percentagem de pais e mães com formação superior $\left(\chi^{2}(2)=12,47, p<0,01 ; \chi^{2}(2)=9,55, p<\right.$ 0,01 , para pais e mães respectivamente). Todos os participantes eram brancos e podiam ser considerados indivíduos saudáveis, tendo apenas sofrido doenças mais comuns e de duração limitada no tempo, tais como a gripe ou as doenças típicas de infância.

\section{Instrumentos}

Aos participantes foi administrado um conjunto de instrumentos que procuravam operacionalizar os seguintes conceitos: (a) Otimismo Comparativo. Foi utilizado um método indireto de operacionalização (e.g., Armor \& Taylor, 1998), no qual cada participante teria de efetuar os julgamentos de risco separadamente para si próprio e para um alvo determinado. A escolha deste método possibilitou, por um lado, a discriminação do peso relativo dos julgamentos para o próprio e para o alvo no OC. Por outro lado, permitiu a aplicação do instrumento a participantes mais novos que, por haver a possibilidade de ainda não conseguirem coordenar simultaneamente duas dimensões, poderiam ver as suas respostas afetadas pela utilização de um método direto em que os julgamentos são efetuados para o próprio em comparação com outros.

Foram escolhidos oito acontecimentos prejudiciais para a saúde: gripe, otite, pneumonia, asma, partir um braço, câncer, aids e ataque de coração. A escolha destes acontecimentos esteve assente nos resultados de um estudo exploratório que visou à análise do conhecimento de crianças e adolescentes portugueses sobre a variedade de doenças existentes (Bernardes, 2002; estudo 1). Numa primeira fase, os participantes efetuavam os julgamentos de risco face ao próprio e para cada acontecimento respondendo à seguinte questão: "Você acha que no seu futuro (ex., nos próximos 10 anos) pode vir a ter...”. No fundo da página era apresentada uma escada com nove degraus que visava representar visualmente uma escala de resposta com nove pontos e cinco âncoras: 1 (Não vou ter com certeza), 3 (Acho que não vou ter), 5 (Tanto posso ter como não ter), 7 (Acho que vou ter) e 9 (Vou ter com certeza). Os participantes deveriam colocar um círculo ao redor do número que mais bem correspondesse à sua resposta. Mais tarde, era-lhes pedido para pensarem "num(a) jovem da mesma idade e do mesmo sexo que você já tenha visto (ex., na rua, no seu bairro, na praia,...) mas que não o (a) conheça (ex., nunca tenha falado ou estado com ele/ a)”. Assim, posteriormente à apresentação do alvo, os sujeitos teriam que efetuar os julgamentos de risco, para cada acontecimento, respondendo à mesma questão acima mencionada mas relativamente ao alvo em causa, ou seja, "Você acha que no futuro (ex., nos próximos 10 anos) esse(a) jovem pode vir a ter...”. A escala de resposta era em tudo semelhante à anterior, com exceção de as âncoras estarem formuladas na 3a pessoa. A diferença entre os julgamentos de risco para o próprio e para o alvo permitiu determinar a intensidade do OC face aos diferentes eventos. Tais índices possuíram uma amplitude de -8 (pessimismo comparativo) a +8 (otimismo comparativo). Foi construído um indicador geral do enviesamento otimista a partir da média dos oito índices de OC ( $\alpha=0,63$ e 0,78 , para pré-adolescentes e adolescentes respectivamente).

(b) Percepção Absoluta e Comparativa de Controle. A par dos julgamentos de risco era pedido aos participantes que efetuassem julgamentos relativamente ao controle que, tanto os próprios como o alvo, possuíam sobre cada um dos acontecimentos acima descritos. Desta forma, em primeiro lugar, os participantes deveriam responder à questão "Você acha que consegue evitar ter ...”, mais uma vez numa escala 
de nove pontos com cinco âncoras: 1 (Com certeza que não consigo evitar), 3 (Acho que não consigo evitar), 5 (Tanto posso conseguir como não conseguir evitar), 7 (Acho que consigo evitar), 9 (Com certeza que consigo evitar). A resposta a esta questão, quando considerada individualmente e em termos absolutos permitiu operacionalizar a percepção absoluta de controle que o indivíduo possuía sobre cada um dos acontecimentos. Posteriormente, após a indução do alvo de comparação já mencionado, o participante era levado a responder à questão "Você acha que esse(a) jovem consegue evitar...", na mesma escala embora com as âncoras formuladas na $3^{\text {a }}$ pessoa. Foi a diferença entre os julgamentos de controle para o próprio e para o alvo que permitiu determinar os índices de percepção comparativa de controle face aos diferentes eventos. Tais índices possuíam uma amplitude de -8 (tenho menos controle que o alvo) a +8 (tenho mais controle que o alvo). A média dos oito índices permitiu constituir um indicador geral de percepção comparativa de controle ( $\alpha=0,58$ e 0,77 , para pré-adolescentes e adolescentes, respectivamente).

(c) Experiência com os eventos. De forma a avaliar a experiência pessoal dos indivíduos com cada um dos acontecimentos descritos, e adaptando a forma de operacionalização utilizada por Weinstein (1980), foi apresentada uma tabela na qual o indivíduo poderia escolher uma ou mais das seguintes alternativas para cada um dos eventos: "Já tive”, “Conheço pessoas que já tiveram”, "Não conheço ninguém que tenha tido”. Da análise das suas respostas os indivíduos foram ordenados em função da sua experiência passada com cada um dos eventos na seguinte escala: (1) Não conhece ninguém que tenha tido, (2) Conhece pessoas que já tiveram, (3) Já teve e (4) Já teve e conhece pessoas que já tiveram.

\section{Procedimento}

Para ambos os grupos etários a aplicação dos instrumentos foi feita em grupo; no entanto, enquanto que para os mais velhos apenas as instruções iam sendo lidas em voz alta, para os mais novos todo o processo de preenchimento era acompanhado por tal leitura. Ainda, os grupos dos préadolescentes possuíam sensivelmente metade do tamanho $(M=10)$ que os grupos de adolescentes. Numa primeira parte, os participantes efetuaram os julgamentos de risco, seguidos dos julgamentos de controle face ao próprio. Antes de cada tipo de julgamento eram transmitidas instruções específicas ao grupo sobre a tarefa em questão, procurando a investigadora certificar-se de que todos compreendiam a escala de resposta. Numa segunda fase, após a recolha do material já preenchido, os participantes efetuaram os julgamentos de risco, seguidos dos julgamentos de controle, desta vez relativamente ao alvo. Numa terceira parte, os indivíduos procederam ao preenchimento da tabela referente à experiência pessoal passada relativamente a cada um dos eventos. Finalmente, era recolhida a informação sóciodemográfica. O tempo de aplicação para ambos os grupos variou entre 15 a 20 minutos.

\section{Resultados}

\section{Diferenças etárias no otimismo comparativo}

Antes de averiguarmos a existência de diferenças etárias no OC, procuramos analisar em que medida tal fenômeno se expressou em cada grupo etário. Assim, de forma a verificar se os valores médios dos oito índices de OC diferiam significativamente de zero, foi realizada uma série de testes $t$ Student para uma amostra. Concluiu-se que, independentemente do grupo etário, e para todos os eventos com exceção da gripe, as médias das distribuições foram significativamente superiores a zero ( $p<0,01$; Figura 1). Estes resultados comprovaram a tendência dos participantes para se verem em média como mais invulneráveis aos diferentes acontecimentos que o alvo de comparação, ou seja, para serem comparativamente otimistas.

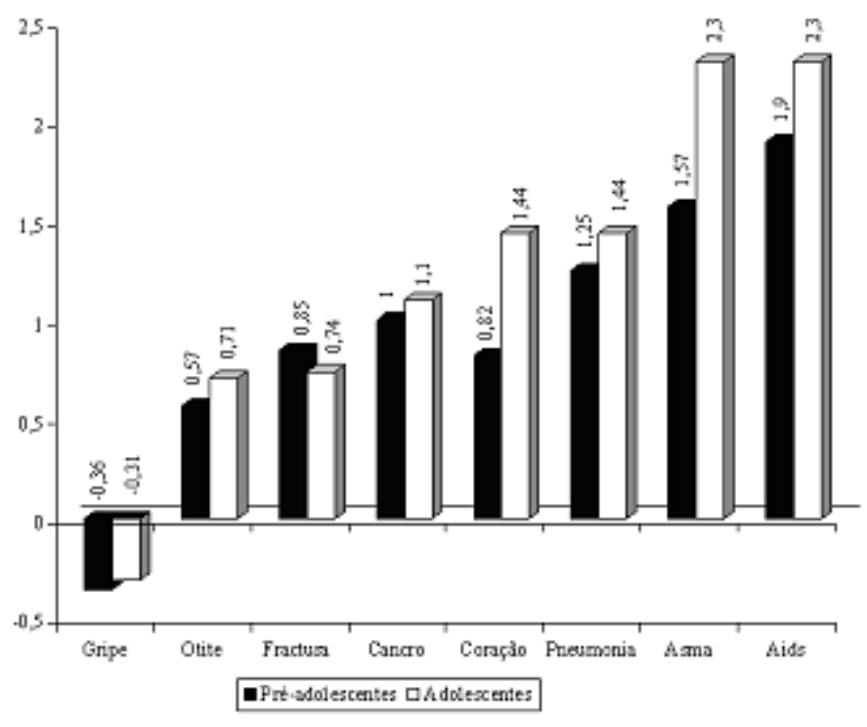

Figura 1. Valores médios dos índices de Otimismo Comparativo para cada grupo etário.

Uma análise de variância multivariada com medidas repetidas foi realizada no sentido de explorar a existência de possíveis diferenças na intensidade do OC entre os participantes dos diferentes grupos etários. Tal análise realizou-se tendo como fator intra-sujeitos os índices de OC para cada acontecimento (8) e o grupo etário (2) como fator inter-sujeitos. Análises preliminares permitiram-nos constatar que, tanto o nível de escolaridade da mãe, como a experiência passada com as doenças não apresentaram efeitos significativos sobre o enviesamento otimista, pelo que tais variáveis não foram consideradas em análises posteriores. Desta forma, constatou-se, em primeiro lugar, que a magnitude do enviesamento otimista varia com o tipo de acontecimento $\left(F_{(7,1253)}=30,47, p=0,00\right.$; Figura 1$)$. Testes post-hoc Scheffé salientaram que o enviesamento otimista é mais intenso face à aids e a asma e menos intenso face à otite, fratura, câncer e ataque de coração $(p<0,01)$. Em geral, não se encontraram 
diferenças etárias no OC, dado que tanto o efeito principal do grupo etário $\left(F_{(1,179)}=0,95, p=0,33\right)$ como o efeito de interação $\left(F_{(7,1253)}=0,98, p=0,45\right)$ não se mostraram significativos.

\section{Diferenças etárias nas Percepções Comparativas de Controle}

À semelhança do procedimento realizado anteriormente, de forma a averiguar se os valores médios dos índices das percepções comparativas de controle diferiam significativamente de zero foi realizada uma série de testes $t$ Student para uma amostra (Figura 2). Constatou-se que todos os participantes se perceberam como tendo mais controle que o alvo de comparação sobre a pneumonia, asma e aids $(p<0,01)$. Além disso, os mais novos mostraram possuir tal enviesamento sobre as fraturas e o câncer $(p<0,05)$, enquanto os mais velhos o apresentaram face aos ataques de coração $(p<0,01)$. Assim, de uma maneira geral, constatou-se que face a alguns dos acontecimentos parece existir um enviesamento nas próprias percepções de controle favorecendo o próprio em detrimento do outro.

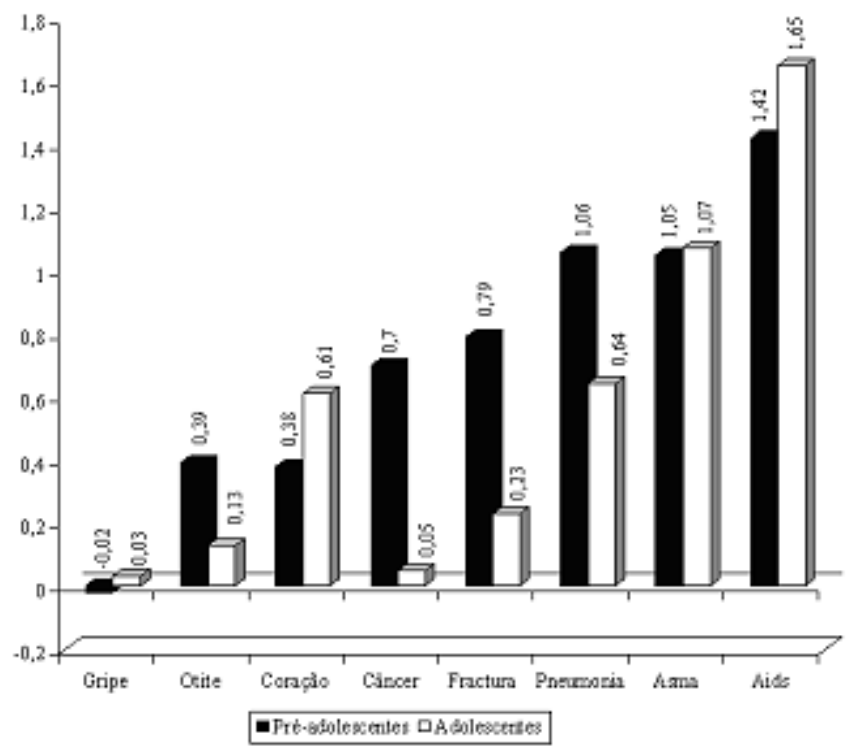

Figura 2. Valores médios dos índices de Percepção Comparativa de Controle para cada grupo etário.

Uma análise de variância multivariada com medidas repetidas foi realizada no sentido de explorar a existência de possíveis diferenças nas percepções comparativas de controle entre os participantes dos diferentes grupos etários. Tal análise foi realizada tendo como fator intra-sujeitos os índices de percepção comparativa de controle para cada acontecimento (8) e o grupo etário (2) como fator inter-sujeitos. Novamente, é de referir que análises preliminares nos permitiram constatar que, tanto o nível de escolaridade da mãe, como a experiência passada com as doenças não apresentaram efeitos significativos sobre tais percepções de controle, pelo que estas variáveis não foram consideradas em análises posteriores. Assim, verificou-se em primeiro lugar que, tal como o OC, a magnitude das percepções comparativas de controle varia com o tipo de acontecimento $\left(F_{(7,1232)}=8,33\right.$, $p=0,00)$. Por um lado, não se encontraram diferenças significativas entre o próprio e o alvo para a gripe e otite, por outro lado, os valores mais elevados de enviesamento encontram-se face à aids, asma e pneumonia (teste post-hoc Scheffé para $p<0,00$ ). Perante a ausência de significância do efeito principal de grupo etário $\left(F_{(1,16)}=0,72, p=0,40\right)$ e do efeito de interação $\left(F_{(7,1232)}=1,28, p=0,26\right)$, concluiu-se que não se constatam diferenças etárias nas percepções comparativas de controle.

\section{Percepções de Controle como preditoras do Otimismo Comparativo}

A partir dos indicadores gerais acima descritos, procurouse avaliar qual o valor preditivo das percepções absolutas e comparativas de controle sobre o OC. Para tal realizaram-se procedimentos de regressão múltipla hierárquica em que, num primeiro modelo, foi apenas introduzido isoladamente o indicador de percepção absoluta de controle e, num segundo modelo, era acrescentado o indicador de percepção comparativa de controle. Este procedimento foi efetuado separadamente para cada uma das sub-amostras (Tabela 1).

Tabela 1

Regressão múltipla hierárquica das Percepções Absolutas (PAC) e Comparativas (PCC) de Controle sobre o Otimismo Comparativo

\begin{tabular}{lcccccc}
\hline \multirow{2}{*}{ Preditores } & \multicolumn{3}{c}{ Pré-Adolescentes } & \multicolumn{3}{c}{ Adolescentes } \\
\cline { 2 - 7 } & $R^{2}$ & $\Delta R^{2}$ & $\beta$ & $R^{2}$ & $\Delta R^{2}$ & $\beta$ \\
\hline Modelo 1 & & & & & \\
PAC & 0,03 & 0,03 & 0,17 & $0,07^{*}$ & $0,07^{*}$ & $0,27^{*}$ \\
Modelo 2 & & & & & & \\
PAC & \multicolumn{5}{c}{$-0,10$} \\
PCC & $0,19^{* *} 0,16^{* *}$ & $0,48^{* *}$ & $0,30^{* *}$ & $0,23^{* *}$ & $0,61^{* *}$ \\
\hline
\end{tabular}

${ }^{*} p<0,01 ; * * p<0,001$

Analisando os resultados para os pré-adolescentes, verificou-se em primeiro lugar que as percepções absolutas de controle não têm qualquer valor preditivo sobre o OC. Foram apenas as percepções comparativas de controle que, quando introduzidas no modelo, explicaram cerca de $16 \%$ da variância do enviesamento otimista $\left(F_{(1,82)}=16,22, p<0,00\right)$. Já no que diz respeito aos adolescentes, constatou-se que as percepções absolutas de controle contribuem, embora modestamente, para a predição do OC $\left(F_{(1,87)}=6,96, p=0,01\right)$. Todavia, quando as percepções comparativas de controle foram introduzidas no modelo aumentaram substancialmente a variância explicada $\left(F_{(1,86)}=28,12, p=0,00\right)$. Efetivamente, as percepções comparativas de controle passaram a ser o único preditor com peso significativo no modelo, eliminando mesmo a variância explicada pelas percepções absolutas de controle. Sabendo em simultâneo que as percepções absolutas de controle explicaram cerca de $37 \%$ da variância das percepções comparativas de controle neste grupo etário $\left(R^{2}=0,37\right.$; 
$\left.F_{(1,91)}=52,87, p=0,00 ; \beta=0,61, p=0,00\right)$, podemos afirmar que estas últimas pareceram mediar totalmente a relação entre as percepções absolutas de controle e o enviesamento otimista (Baron \& Kenny, 1986).

\section{Discussão}

Previamente à análise das diferenças etárias no OC, procurou-se averiguar a existência de tal enviesamento face à saúde na adolescência. Este estudo permitiu-nos constatar que, tanto pré-adolescentes como adolescentes, possuem uma percepção comparativa de invulnerabilidade face à quase totalidade dos eventos apresentados. Para ambos os grupos etários, e para todos os eventos com exceção da gripe, as diferenças entre os julgamentos face ao próprio e face ao alvo foram significativamente superiores a zero. Estes dados vêm reforçar as escassas evidências que mais recentemente se encontram na literatura e que, também elas, mostram a prevalência deste fenômeno entre os mais jovens (Arnett, 2000; Chapin et al., 2005; Cohn et al., 1995; Gladis et al., 1992; Quadrel et al., 1993; Whalen et al., 1994).

Todavia, pensamos estar longe de poder afirmar a existência de uma percepção de risco global dado que, à semelhança do encontrado por outros autores (Quadrel et al., 1993; Whalen et al., 1994), também pré-adolescentes e adolescentes parecem possuir percepções de risco específicas em função do evento em causa. Assim, de uma maneira geral, foram doenças como a asma e a aids, que despertaram percepções de invulnerabilidade mais intensas. É de salientar ser a aids uma doença face à qual tipicamente se têm encontrado as percepções de invulnerabilidade mais elevadas em crianças e adolescentes (Cohn et al., 1995; Gladis et al., 1992; Whalen et al., 1994). Tal poderá estar em parte associado à existência de um estereótipo de vítima de tal enfermidade que, estando acessível, permite ao indivíduo uma maior diferenciação entre o seu próprio risco de contrair a doença e o de um alvo (Weinstein, 1980). As elevadas percepções de invulnerabilidade comparativa face a tal enfermidade na adolescência são preocupantes. Sendo a adolescência uma fase de descoberta da vida sexual, tais percepções de invulnerabilidade poderão conduzir a comportamentos sexuais de risco. Efetivamente, Gladis e colaboradores (1992) mostraram a presença de uma discrepância entre os julgamentos de risco absolutos e comparativos face à aids e o envolvimento real dos adolescentes em comportamentos de risco. Por este motivo, parece-nos fundamental a clarificação do papel que tais percepções poderão desempenhar na determinação de tais comportamentos e, conseqüentemente, na propagação de uma doença que atualmente assume proporções epidêmicas. Em contraste, além da já mencionada ausência de otimismo face à gripe, são os eventos como a otite, a fratura de um braço, o câncer e o ataque de coração que despertam um OC menos intenso. Estes resultados são muito semelhantes aos encontrados por Whalen e colaboradores (1994) com pré-adolescentes. Também, confirmando evidências recolhidas por outros autores (Cohn et al., 1995; Whalen et al., 1994), constatou-se que a gripe, provavelmente pela sua elevada prevalência e reduzidas conseqüências, foi um evento face ao qual os indivíduos não demonstraram qualquer tipo de enviesamento. Estes fatores poderão igualmente justificar o reduzido enviesamento face à otite e fratura do braço. Já no que diz respeito ao câncer a ao ataque de coração, na generalidade, os participantes perceberam tais eventos como menos controláveis que os restantes. A percepção de menor controlabilidade dos eventos poderá ter contribuído para a redução do OC.

Todavia, apesar da presença de tal enviesamento entre os participantes, esteve patente nos resultados a semelhança na forma como pré-adolescentes e adolescentes entendem o risco comparativo face aos eventos apresentados. As diferenças etárias na intensidade dos julgamentos de risco comparativos estiveram praticamente ausentes. Estes resultados contrastam com evidências de um estudo recente realizado com adolescentes entre os 13 e os 18 anos, que salienta que a idade dos mesmos estava positivamente relacionada com a intensidade do OC face ao fato de serem vítimas de violência (Chapin et al., 2005). Todavia, a comparação direta com tais evidências deve ser feita de forma cautelosa, dado que a natureza dos eventos alvo de julgamentos de risco é inerentemente distinta. Assim, o reduzido número de estudos que analisam as diferenças etárias no OC deixanos sem a possibilidade de enquadramento empírico e teórico dos presentes resultados. Aparentemente, a forma como pré-adolescentes e adolescentes entendem o risco de forma comparativa face às diferentes enfermidades operacionalizadas é muito semelhante.

Já no que diz respeito às percepções comparativas de controle, os resultados permitem-nos afirmar que embora os participantes se tenham percebido como tendo mais controle que um jovem do mesmo sexo e da mesma idade face a certos eventos, mais uma vez as diferenças etárias primaram pela ausência. A pneumonia, asma e aids foram doenças que todos os participantes julgaram poder melhor controlar que o alvo de comparação. Além destas, os préadolescentes perceberam-se como tendo mais controle que o alvo sobre o câncer e as fraturas, enquanto os mais velhos mostraram tal enviesamento face aos ataques cardíacos. Todavia, a análise dos indicadores gerais das percepções comparativas de controle mostrou não existirem quaisquer diferenças etárias neste tipo de percepções. Estes resultados vão de encontro aos dados obtidos por Quadrel e colaboradores (1993), que salientam que face a diversos eventos ameaçadores para a saúde os adolescentes apresentam percepções de controle enviesadas a seu favor. Tal como para o OC, o enviesamento nas percepções comparativas de controle pareceu variar em função do acontecimento em causa. Novamente, a aids está entre os acontecimentos que 
despertam enviesamentos mais intensos, enquanto a gripe e otite se encontram entre aqueles que induzem menor enviesamento. Tal semelhança com os resultados do OC, poderia levar-nos a concluir que as percepções comparativas de risco e controle partilham alguns fatores determinantes, tais como, a prevalência dos acontecimentos ou o seu grau de estigmatização. Todavia, tal conclusão seria precipitada tendo em conta que ainda muito está por fazer, particularmente no que diz respeito ao estudo dos determinantes das percepções comparativas de controle. Pensamos que estudos futuros possam clarificar os motivos pelos quais determinados eventos despertam com maior intensidade tal tipo de enviesamento. A clarificação dos fatores que, subjacentes às representações de cada tipo de doença, possam contribuir para tais percepções comparativas de controle parece-nos poder ser relevante para o trabalho com adolescentes que padeçam de tais enfermidades.

No que diz respeito ao terceiro objetivo do presente estudo, verificou-se que os julgamentos absolutos e comparativos de controle parecem desempenhar diferentes papéis na predição do OC em função do grupo etário. Em primeiro lugar, constatou-se que, em ambos os grupos etários, as percepções comparativas de controle possuem um maior poder preditivo do OC que as percepções absolutas de controle. Aliás, entre os pré-adolescentes a noção subjetiva de poder controlar pessoalmente um determinado evento não mostrou ter qualquer relação com o OC. De fato, estes resultados vêm confirmar as suspeitas de Harris (1996), ao avançar a hipótese de serem os julgamentos comparativos de controle que melhor determinam o enviesamento otimista. Entre os adolescentes mais velhos, embora os julgamentos absolutos de controle tenham tido algum impacto sobre o OC, verificou-se que tal efeito foi inteiramente mediado pelas percepções comparativas de controle. Estes resultados contradizem o vasto corpo empírico que aponta para uma relação consistente entre a percepção de controle e o OC entre os adultos (Harris, 1996; Harris \& Middleton, 1994; McKenna, 1993; Helweg-Larsen \& Shepperd, 2001; Taylor et al., 1992; Van der Velde et al., 1991). Embora tendencialmente apontem para que com a idade tais percepções comparativas assumam maior centralidade na determinação do OC, entre os adolescentes mais velhos o seu poder preditivo permanece reduzido. Efetivamente, tais resultados remetem para primeiro plano o papel que a imagem do outro e os processos de comparação social podem ter ao nível das percepções comparativas de invulnerabilidade. Este dado poderá ser fundamental para o desenvolvimento de programas de promoção da saúde e prevenção da doença dirigidos a essa faixa etária. O impacto negativo que o OC poderá ter ao nível dos comportamentos de prevenção poderá ser minimizado através da implementação de programas que não se centrem exclusivamente no desenvolvimento de comportamentos específicos de saúde, com vistas a aumentar a percepção absoluta de controle sobre uma certa doença. O trabalho sobre a imagem do próprio adolescente relativamente à representação que este possui do seu grupo de pares pode ser fundamental. Concretamente, e a título de exemplo, para além do conhecimento sobre como evitar comportamentos sexuais de risco de forma a prevenir a contração de doenças sexualmente transmissíveis, poderá ser importante, a desconstrução da imagem de invulnerabilidade do próprio relativamente à imagem do seu grupo de pares.

Embora o presente estudo constitua um avanço na compreensão do fenômeno do otimismo comparativo na adolescência, apresenta algumas limitações que deverão ser salientadas. Em primeiro lugar, o fato de a amostra ser constituída por jovens brancos e na sua maioria de um estatuto socioeconômico mediano, poderá ocultar enviesamentos culturais/educacionais. Tanto o nível educacional, como determinadas significações de saúde específicas de determinados grupos étnicos, poderão formatar as percepções de risco e controle. O presente estudo não permite averiguar a presença de tais diferenças. Estudos futuros deverão, ou incluir amostras mais heterogêneas em termos de estatuto socioeconômico/ cultural, ou mesmo, procurar averiguar a presença de tais diferenças. Em segundo lugar, as diferentes formas de administração dos questionários aos diferentes grupos etários poderá levantar questões relativas ao erro introduzido pela variação metodológica. De fato, a forma de administração do instrumento aos pré-adolescentes teve como objetivo minimizar o efeito da capacidade de leitura na compreensão da tarefa pedida. No entanto, a aplicação dos questionários foi efetuada por uma investigadora independente de forma a minimizar a possibilidade de enviesamentos de resposta causados pela mesma. Em terceiro lugar, qualquer estudo sobre diferenças etárias deverá ser interpretado com cuidado no que toca ao seu significado em termos desenvolvimentistas. A idade por si só não é um indicador de desenvolvimento fidedigno. É possível que entre os grupos etários aqui apresentados se possam encontrar participantes que possuam grandes diferenças no que toca ao nível de desenvolvimento das conceptualizações sobre saúde e doença. Pensamos, aliás, que tal fato possa ser uma hipótese explicativa da ausência de diferenças etárias no OC e nas percepções comparativas de controle. De fato, concordamos com Whalen e colaboradores (1994), ao afirmarem que a ausência de diferenças etárias no OC não implica uma semelhança nos processos que lhe estão subjacentes. No caso concreto do presente estudo, embora não se tenham constatado diferenças etárias ao nível dos julgamentos comparativos de risco e controle, uma análise mais apurada dos fatores determinantes de tais enviesamentos nas diferentes fases de desenvolvimento poderá mostrar-se enriquecedora. Todavia, se por um lado, podemos hipotetizar que diferentes conceptualizações de saúde e doença possam afetar julgamentos de risco e controle, por outro, o estudo das diferenças etárias não nos permite testar tal hipótese. A 
relação entre conceptualizações de saúde e doença e as percepções de risco e controle comparativas poderão constituir futuramente uma linha de investigação interessante e profícua.

Mais ainda, a semelhança encontrada na expressão do OC ao longo da adolescência faz-nos refletir sobre a importância não só de um esforço continuado na exploração das diferenças etárias nos determinantes de tal fenômeno, mas também nas suas conseqüências para a saúde dos indivíduos. Embora a temática das conseqüências de tal fenômeno ilusório ainda desperte muita celeuma, a presença de OC em idades tão jovens deverá pelo menos fazer-nos refletir sobre a forma como programas de prevenção são desenhados e implementados, ou mesmo sobre qual a relação entre tais percepções e os comportamentos de prevenção face à saúde que se pretendem ensinar às camadas mais jovens. Pensamos ser de todo o interesse dar continuidade a este projeto de exploração do OC ao longo do desenvolvimento, direcionando-o em particular para as idades mais jovens. A exploração do OC em crianças com menos de 10 anos é virtualmente nula o que, pelas características de tal fase de desenvolvimento, se nos afigura um projeto aliciante. Ainda, apesar de as nossas amostras serem constituídas por indivíduos saudáveis, parece-nos que o estudo comparativo deste fenômeno com populações com doenças crônicas poderá ser um importante avanço para a compreensão desenvolvimentista do fenômeno de adaptação a situações vitimizantes.

\section{Referências}

Armor, D., \& Taylor, S. (1998). Situated optimism: specific outcome expectancies and self regulation. Advances in Experimental Social Psychology, 30, 309-375.

Arnett, J. (2000). Optimistic bias in adolescent and adult smokers and nonsmokers. Addictive Behaviours, 25, 625-632.

Baron, R., \& Kenny, D. (1986). The moderator-mediator variable distinction in social psychological research: conceptual, strategic and statistical considerations. Journal of Personality and Social Psychology, 51, 1173-1182.

Barrio-Martinez, C. (1990). La comprensión infantil de la enfermedad. Madri: Anthrophos.

Bernardes, S. (2002). O optimismo comparativo face à saúde em crianças e adolescentes: diferenças etárias na sua expressão e relação com crenças associadas ao controlo. Dissertação de mestrado não-publicada, Instituto Superior das Ciências do Trabalho e da Empresa, Lisboa.

Beyth-Marom, R., Austin, L., Fischoff, B., Palmgren, C., \& Quadrel, M. (1993). Perceived consequences of risky behaviours: adults and adolescents. Developmental Psychology, 29, 549-563.

Burger, J., \& Burns, L. (1988). The illusion of unique invulnerability and the use of effective contraception. Personality and Social Psychology Bulletin, 14, 264-270.

Chapin, J. (2000). Third-person perception and optimistic bias among urban minority at-risk youth. Communication Research, 27, 51-81.
Chapin, J., de las Alas, S., \& Coleman, G. (2005). Optimistic bias among potential perpetrators and victims of youth violence. Adolescence, 40, 749-760.

Chapin, J., \& Gleason, D. (2004). Student perceptions of school violence: could it happen here? Journal of Adolescent Research, 19, 360-376.

Clarke, V., Williams, T., \& Arthey, S. (1997). Skin type and optimistic bias in relation to the sun protection and sun tanning behaviors of young adults. Journal of Behavioral Medicine, 20, 207-222.

Cohn, L., Macfarlane, S., Yanez, C., \& Imai, W. (1995). Risk perception: differences between adolescents and adults. Health Psychology, 14, 217-222.

Elkind, D. (1967). Egocentrism in adolescence. Child Development, 38, 1025-1034.

Fontaine, K., \& Smith, S. (1995). Optimistic bias in cancer risk perception: a cross-national study. Psychological Reports, 77, 143-146.

Gladis, M., Michela, J., Walter, H., \& Vaughan, R. (1992). High school students' perceptions of AIDS risk: realistic appraisal or motivated denial? Health Psychology, 11, 307-316.

Harris, P. (1996). Sufficient grounds for optimism? The relationship between perceived controllability and optimistic bias. Journal of Social and Clinical Psychology, 15, 9-52.

Harris, P., \& Middleton, W. (1994). The illusion of control and optimism about health: on being less at risk but no more in control than others. British Journal of Social Psychology, 33, 369-386.

Helweg-Larsen, M., \& Shepperd, J. (2001). Do moderators of the optimistic bias affect personal or target risk estimates? A review of the literature. Personality and Social Psychology Review, 5, 74-95.

Kershaw, T., Ethier, K., Niccolai, L., Lewis, J., \& Ickovics, J. (2003). Misperceived risk among female adolescents: social and psychological factors associated with sexual risk. Health Psychology, 22, 523-532.

Klein, C., \& Helweg-Larsen, M. (2002). Perceived control and the optimistic bias: a meta-analytic review. Psychology \& Health, 17, 437-446.

Kos, J., \& Clarke, V. (2001). Is optimistic bias influenced by control or delay? Health Education Research, 16, 533-540.

McKenna, F. (1993). It won't happen to me: unrealistic optimism or illusion of control? British Journal of Psychology, 84, 39-50.

McKenna, F., \& Albery, I. (2001). Does unrealistic optimism change following a negative experience? Journal of Applied Social Psychology, 31, 1146-1157.

McKenna, F., Stainer, R., \& Lewis, C. (1991). Factors underlying illusory selfassessment of driving skill in males and females. Accident Analysis and Prevention, 23, 45-52.

Millstein, S. (1993). Perceptual, attributional and affective processes in perceptions of vulnerability through the life span. In N. Bell \& R. Bell (Orgs.), Adolescent risk taking (pp. 55-65). California: Sage.

Moen, B., \& Rundmo, T. (2005). Predictors of unrealistic optimism: a study of Norwegian risk takers. Journal of Risk Research, 8, 363-382.

Perrin, E., \& Gerrity, P. (1981). There's a demon in your belly: children's understanding of illness. Pediatrics, 67, 841-849.

Quadrel, M., Fischoff, B., \& Davis, W. (1993). Adolescent (in)vulnerability. American Psychologist, 48, 102-116.

Redmond, E., \& Griffith, C. (2004). Consumer perceptions of food safety risk, control and responsibility. Appetite, 43, 309-313.

Segerstrom, S., Taylor, S., Kemeny, M., \& Fahey, J. (1998). Optimism is associated with mood, coping and immune change in response to stress. Journal of Personality and Social Psychology, 74, 1646-1655.

Sheer, V., \& Cline, R. (1994). The development and validation of a model explaining sexual behaviour among college students: Implications for AIDS communication campaign. Human Communication Research, 21, 280-304. 
Taylor, S., Kemeny, M., Aspinwall, L., Schneider, R., Rodriguez, R., \& Herbert, M. (1992). Optimism, coping, psychological distress and high-risk sexual behaviour among men at risk for acquired immunodeficiency syndrome (AIDS). Journal of Personality and Social Psychology, 63, 460-473.

Thompson, S., Armstrong, W., \& Thomas, C. (1998). Illusions of control, underestimations and accuracy: a control heuristic explanation. Psychological Bulletin, 123, 143-161.

Van der Velde, F., Hooykaas, C., \& Van Der Pligt, J. (1991). Risk perception and behaviour: pessimism, realism and optimism about aids-related health behaviour. Psychology and Health, 6, 1-16.
Weinstein, N. (1980). Unrealistic optimism about future life events. Journal of Personality and Social Psychology, 39, 806-820.

Weinstein, N. (1987). Unrealistic optimism about susceptibility to health problems: conclusions from a community-wide sample. Journal Behavioral Medicine, 10, 11-20.

Weinstein, N., \& Klein, W. (1996). Unrealistic optimism: present and future. Journal of Social and Clinical Psychology, 15, 1-8.

Whalen, C., Henker, B., O’Neil, R., Hollingshead, J., Holman, A., \& Moore, B. (1994). Optimism in children's judgements of health and environmental risks. Health Psychology, 13, 319-325.

Sónia F. Bernardes, mestre em Psicologia Social e Organizacional pelo Instituto Superior das Ciências do Trabalho e da Empresa (ISCTE, Portugal), é Professora Assistente no Departamento de Psicologia Social e das Organizações do Instituto Superior das Ciências do Trabalho e da Empresa. Endereço para correspondência: Av. das Forças Armadas (cacifo 34 AA) 1649-026. Lisboa, Portugal. Tel.: ** 35191 778-2005. Fax: ** 35121 790-3002. E-mail: sonia.bernardes@iscte.pt

Maria Luísa Lima, doutora em Psicologia Social e Organizacional pelo Instituto Superior das Ciências do Trabalho e da Empresa (ISCTE, Portugal), é Professora Associada com Agregação no Departamento de Psicologia Social e das Organizações do Instituto Superior das Ciências do Trabalho e da Empresa. 